\title{
FINER THEORETICAL ASPECTS OF THE DRIFT FLUX FAMILY OF EQUATIONS FOR SIMULATING AVERAGED VOLUME FRACTION IN MULTIPHASE FLOWS
}

\author{
A. S. NAGOO \& M. M. SHARMA
}

Department of Petroleum and Geosystems Engineering, The University of Texas, Austin, USA.

\begin{abstract}
The drift flux formulation is widely regarded as being amenable to computationally efficient calculation of averaged slip between phases (i.e. averaged volume fraction) in multiphase flows. However, contrary to the common perception in practice that this formulation provides two simple data-fitting parameters for describing averaged slip, it is shown that these parameters cannot be mathematically disassociated from each other and are intricately related to both the averaged and local phase velocities and volume fractions. This derived fact also provides a rational explanation for the extreme difficulty in the past to resolve the influence of the local lateral distribution (multidimensional) effects of the dispersed phase in the flow on the averaged flow characteristics. Of further significance from this derivation is the fact that correlations for drift flux parameters in which these terms are expressed explicitly from each other cannot claim a better understanding of the flow physics unless they capture this inherent analytical bidirectional dependency. All of the multiphase flow variables that affect the drift velocity parameter also affect the distribution parameter. In terms of simulation applications, the new analytical derivations presented enable a re-formulation and unification of prior volume fraction models and an explanation for field observations of why a reduction in the drift velocity parameter will lead to reduction in the distribution parameter. As much as numerical computing and simulation methods are invaluable tools, the results of this work show that it is equally important to explore their mathematical basis and scientific understanding that can help to advance more efficient simulation of multiphase flow behaviors in the field.
\end{abstract}

Keywords: analytical prediction, drift flux parameters, fractional flow theory, multiphase flow simulation, pipe, pipeline, slip ratio, volume fraction, wellbore.

\section{INTRODUCTION}

A large and quite popular family of averaged volume fraction equations are of the drift flux type, originally formalized in Zuber and Findlay [1]. A detailed historical perspective of the drift flux formulation can be found in Appendix A of Chexal and Lellouche [2]. Considering an arbitrary two-phase system consisting of a less dense phase (phase-2) flowing simultaneously with a more dense phase (phase-1), the fundamental starting point for a drift flux representation of this case is a simple algebraic equation for the local phase- 2 velocity, $\vec{v}_{2}$, in terms of a shift (or 'drift') in the local mixture velocity, $\vec{u}_{m i x}$, as noted:

$$
\vec{v}_{2}=\vec{u}_{\text {mix }}+\overbrace{\left(\vec{v}_{2}-\vec{u}_{\text {mix }}\right)}^{\begin{array}{c}
\text { local phase }-2 \\
\text { drift velocity }=
\end{array} \vec{v}_{2, \text { mix }}}
$$

In eqn (1), the local phase-2 drift velocity, $\vec{v}_{2, \text { mix }}$, can be defined in its alternative local relative velocity (or local slip velocity) form, $\vec{v}_{2,1}$, as:

$$
\vec{v}_{2, \text { mix }}=\vec{v}_{2}-\left(\vec{v}_{2} s_{2}+\vec{v}_{1}\left(1-s_{2}\right)\right)=\left(1-s_{2}\right)\left(\vec{v}_{2}-\vec{v}_{1}\right)=\left(1-s_{2}\right) \vec{v}_{2,1}
$$

Where $s_{2}$ is the local phase-2 volume fraction. Now, multiplying eqn (1) throughout by $s_{2}$ to convert from in situ velocities to experimentally measurable volume fluxes, considering only 
the axial components of these velocity vectors and then performing cross-sectional (global) area-averaging, we arrive at:

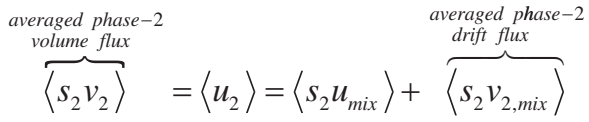

Equation (3) shows the reason why this formulation is referred to as drift flux. Dividing eqn (3) throughout by the product of two directly measurable experimental variables, $\left\langle s_{2}\right\rangle\left\langle u_{\text {mix }}\right\rangle$, we can thus arrive at the familiar form of the drift flux formulation that contains the drift flux parameters, as:

$$
\frac{\left\langle u_{2}\right\rangle}{\left\langle s_{2}\right\rangle}=\overbrace{\left\langle\left\langle v_{2}\right\rangle\right\rangle}^{\begin{array}{c}
\text { averaged phase-2 } \\
\text { velocity }
\end{array}}=\overbrace{\left(\frac{\left\langle s_{2} u_{\text {mix }}\right\rangle}{\left\langle s_{2}\right\rangle\left\langle u_{\text {mix }}\right\rangle}\right)}^{\begin{array}{c}
\text { averaged mixture } \\
\text { distribution parameter }
\end{array}}\left\langle u_{\text {mix }}\right\rangle+\overbrace{\left(\frac{\left\langle s_{2} v_{2, \text { mix }}\right\rangle}{\left\langle s_{2}\right\rangle}\right)}^{\begin{array}{c}
\text { averaged phase-2 } \\
\text { avift velocity parameter } \\
=\left\langle\left\langle v_{2, \text { mix }}\right\rangle\right\rangle
\end{array}}
$$

\subsection{Rationale for fractional flow representation}

The drift flux parameters in eqn (4), it would appear, could conceptually be obtained from a simple linear fit to the graph of $\left\langle\left\langle v_{2}\right\rangle\right\rangle$ versus $\left\langle u_{\text {mix }}\right\rangle$. Indeed, such dimensional plots were common in the early literature (particularly with Russian investigators, [3, 4]) as a means for displaying averaged volume fraction data. However, these plots were found to only work well in experiments where the averaged slip velocity, $S_{2,1}=\left\langle\left\langle v_{2}\right\rangle\right\rangle-\left\langle\left\langle v_{1}\right\rangle\right\rangle$, was low. For low mass flow rates of the more dense phase where $S_{2,1}$ was high, these plots proved problematic to investigators who were seeking a simple straight line correlation fit of their averaged volume fraction data. Indeed, this was the same argument provided by Zuber and Findlay [1].

It would seem during this period a proclivity to correlate experimental data on a straight line was more desirable than (or overlooked) a more flexible and powerful way to explain the inter-relations of averaged volume fraction observations through the dimensionless plot of averaged phase- 2 fractional flow, $f_{2}$, versus $\left\langle s_{2}\right\rangle$, where $f_{2}=\left\langle u_{2}\right\rangle /\left\langle u_{\text {mix }}\right\rangle$ is a ratio of two directly measurable (and known) experimental variables. If averaged volume fraction experimental data are plotted on these fractional flow curves instead of a straight line, then a family of slip ratio curves would result when considering that the sole multiphase flow variable relating $f_{2}$ to $\left\langle s_{2}\right\rangle$ is the dimensionless slip ratio, $H_{2,1}=\left\langle\left\langle v_{2}\right\rangle\right\rangle /\left\langle\left\langle v_{1}\right\rangle\right\rangle$. This fractional flow equation can be derived as:

$$
f_{2}=\frac{\left\langle u_{2}\right\rangle}{\left\langle u_{\text {mix }}\right\rangle}=\frac{\left\langle\left\langle v_{2}\right\rangle\right\rangle\left\langle s_{2}\right\rangle}{\left\langle\left\langle v_{2}\right\rangle\right\rangle\left\langle s_{2}\right\rangle+\left\langle\left\langle v_{1}\right\rangle\right\rangle\left(1-\left\langle s_{2}\right\rangle\right)}=\left(\frac{H_{2,1}}{1+\left(H_{2,1}-1\right)\left\langle s_{2}\right\rangle}\right)\left\langle s_{2}\right\rangle
$$

Equivalently, a re-arrangement of eqn (5) in terms of $\left\langle s_{2}\right\rangle$ would yield:

$$
\left\langle s_{2}\right\rangle=\frac{1}{H_{2,1}\left(\frac{1-f_{2}}{f_{2}}\right)+1}
$$


A further re-arrangement of eqn (6) in terms of $H_{2,1}$ would yield:

$$
H_{2,1}=\left(\frac{1-\left\langle s_{2}\right\rangle}{\left\langle s_{2}\right\rangle}\right)\left(\frac{1-f_{2}}{f_{2}}\right)^{-1}
$$

Although not directly obvious, eqn (5) can be reduced to the fundamental void-quality relation if mass fluxes are used instead of volume fluxes in defining the flow quality. In terms of mathematical self-consistency, we note that the no-averaged-slip condition of $H_{2,1}=1$, correctly reduces eqn (5) to $f_{2}=\left\langle s_{2}\right\rangle$. Also, in eqn (5), at $\left\langle s_{2}\right\rangle=0$ (i.e. no phase-2 present), $f_{2}=0$, and at $\left\langle s_{2}\right\rangle=1$ (i.e. no phase- 1 present), $f_{2}=1$.

In general, as seen in eqn (7) above, $H_{2,1}$ will be a function depending on several multiphase flow parameters through its direct relationship with $\left\langle s_{2}\right\rangle$ and $f_{2}$. Hence, the transition through different $H_{2,1}$ values for any dataset will result in a unique fractional flow path for the dataset. This is the essence of the fractional flow representation of averaged volume fraction data, which characterizes in a general way (and not merely as a data display or data correlation tool), the $\left\langle s_{2}\right\rangle$ behavior with respect to $f_{2}$, the prevailing system operating conditions and the phase properties for an experimental dataset. The real power of fractional flow paths lies in its ability to generate single-path or multiple-path traverses connecting different flow patterns, regardless of whether those paths correlate data on a straight line or not. From a transport phenomena perspective, the objectively measurable changes in the phase velocities and volume fractions associated with each flow pattern are the fundamental physical quantities that govern the transport processes of the multiphase flow. It is these transport processes that drive the different mass, momentum and energy exchanges occurring during flow and this is why flow patterns matter. In the fractional flow representation, flow pattern inter-relationships are neatly captured as different fractional flow paths. Traversing these paths connects the different flow patterns thus capturing the relationships between different flow phenomena.

For plotting purposes, we can view a simplified graphical representation of eqn (5) in limiting terms of constant $H_{2,1}$ iso-slip curves, as shown in Fig. 1. Every averaged volume fraction dataset will transition through a family of these curves (along a fractional flow path) in accordance with the $H_{2,1}$ model for the dataset.

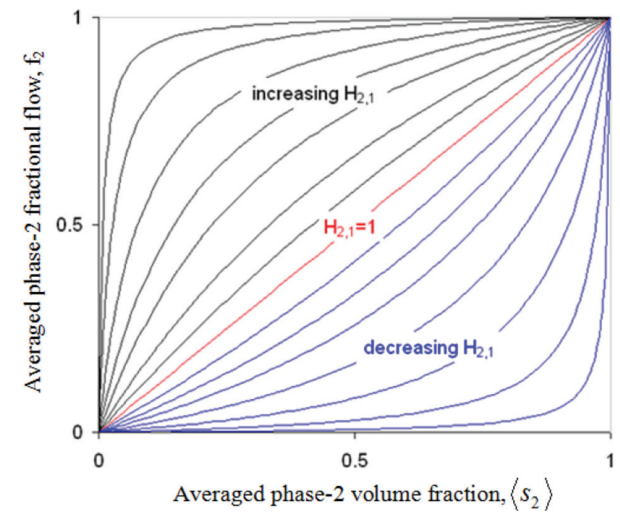

Figure 1: A graphical representation of eqn (5) in simplified (limiting) terms of constant $H_{2,1}$ iso-slip curves. 


\subsection{Drift flux family of equations as slip ratios and slip velocities}

With the fractional flow representation in place, it now becomes clear why the drift flux family of equations are generally applicable to any multiphase flow scenario. Different drift flux models (formed from different scenario-specific drift flux parameters) can be seen as merely different closure relationships for $H_{2,1}$. To see this, we can rearrange eqn (4) as:

$$
\frac{f_{2}}{\left\langle s_{2}\right\rangle}=C_{0}+\frac{\left\langle\left\langle v_{2, \text { mix }}\right\rangle\right\rangle}{\left\langle u_{\text {mix }}\right\rangle}
$$

Then substituting eqn (8) in eqn (7), we arrive at the most basic one-parameter form of all drift flux equations, as:

$$
H_{2,1}=\left(C_{0}+\frac{\left\langle\left\langle v_{2, \text { mix }}\right\rangle\right\rangle}{\left\langle u_{\text {mix }}\right\rangle}\right)\left(\frac{1-\left\langle s_{2}\right\rangle}{1-f_{2}}\right)
$$

As obvious as eqn (9) demonstrates the general applicability of the drift flux parameters for describing averaged volume fraction behavior, it must be noted there are some investigators who incorrectly believe that these parameters can only satisfactorily accommodate a restricted amount of system geometries, operational conditions or fluid properties. As a concrete example in a specific scenario, there can be a gross misunderstanding of the averaged phase- 2 drift velocity parameter, $\left\langle\left\langle v_{2, m i x}\right\rangle\right\rangle$, in horizontal flow, leading some investigators to wrongly set this parameter to zero in this case. Franca and Lahey [5] put this case into proper context in their paper: 'It should be stressed that for horizontal flows the drift velocity, $V_{G j}$, is not normally zero. The fact that many previous authors have assumed it to be zero just highlights their misunderstanding of what the drift velocity represents.'.

In fact, eqn (9) is a lot more important than it first appears since it shows how $H_{2,1}$ is related to the averaged dispersed-phase particle slip and particle-to-particle behaviors. To see this, we note that a generalized form of $\left\langle\left\langle v_{2, \text { mix }}\right\rangle\right\rangle$ is:

$$
\left\langle\left\langle v_{2, \text { mix }}\right\rangle\right\rangle=\frac{\left\langle s_{2} v_{2, \text { mix }}\right\rangle}{\left\langle s_{2}\right\rangle}=\overbrace{\frac{\left\langle\left(1-s_{2}\right) s_{2} v_{2,1}\right\rangle}{\left\langle s_{2}\right\rangle}}^{\text {from eqn. (2) }} \approx \alpha \overbrace{\alpha\left(1-\beta\left\langle s_{2}\right\rangle^{\chi}\right)^{\delta}}^{\begin{array}{c}
\text { averaged } \\
\text { particle-to-particle } \\
\text { parameters }
\end{array}} \overbrace{\left(\frac{\left\langle s_{2} v_{2,1}\right\rangle}{\left\langle s_{2}\right\rangle}\right)}^{\begin{array}{c}
\text { averaged } \\
\text { particle stip } \\
\text { parameter }
\end{array}}
$$

Then, we substitute eqn (10) in eqn (9) to obtain:

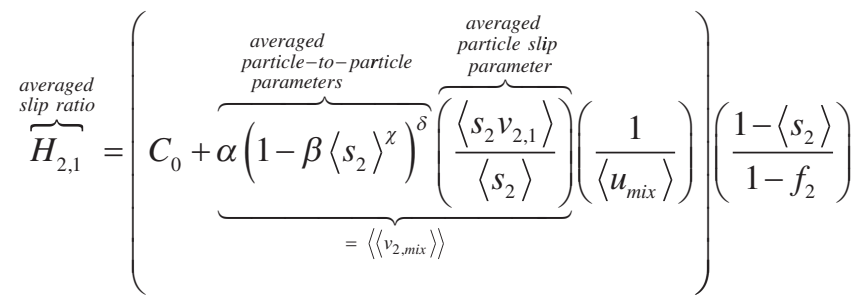

In eqn (11), $C_{0}$, the averaged particle-to-particle parameters $(\alpha, \beta, \chi, \delta)$ and the averaged particle slip parameter can take on different values (or functions of system parameters) for 
various scenarios resulting in a family of drift flux equations. Also, the averaged particle-to-particle term in eqn (11) is alternatively referred to as the hindering function in the literature $[6,7]$.

Equivalently, in place of the averaged slip ratio, $H_{2,1}$, we can re-express eqn (11) in terms of the averaged slip velocity, $S_{2,1}$, as:

$$
\overbrace{S_{2,1}}^{\begin{array}{c}
\text { averaged } \\
\text { slip velocity }
\end{array}}=\left(C_{0}-1\right)\left\langle u_{\text {mix }}\right\rangle+\underbrace{\overbrace{\alpha\left(1-\beta\left\langle s_{2}\right\rangle^{\chi}\right)^{\delta}}^{\delta}}_{=\left\langle\left\langle v_{2, \text { mix }}\right\rangle\right.} \overbrace{\left(\frac{\left\langle s_{2} v_{2,1}\right\rangle}{\left\langle s_{2}\right\rangle}\right)}^{\begin{array}{c}
\text { averaged } \\
\text { particle-to-particle } \\
\text { parameters }
\end{array}}
$$

Equation (12) now clearly shows how the averaged slip velocity of the multiphase flowing mixture could be significantly different from the averaged particle slip parameter. Unfortunately, these variables can sometimes be incorrectly stated by investigators as being equivalent.

Indeed, eqn (12) now explains why it is incorrect to say that $C_{0}=1$, by itself, has a physical meaning associated with phase- 2 being homogenously distributed in phase-1, or, that this condition has any mathematical or physical meaning of $S_{2,1}=0$. Obviously, $C_{0}$, by itself, does not serve as a correction factor to a homogenous flow. Similarly, as seen from eqn (12), $\left\langle\left\langle v_{2, \text { mix }}\right\rangle\right\rangle=0$, by itself, does not mean that $S_{2,1}=0$. In this latter case, non-uniform lateral distributions of phase- 2 in phase- 1 at any pipe angle orientation, could result in $C_{0} \neq$ 1 , and therefore ensure a non-zero $S_{2,1}$ - this is sometimes referred to as integral slip in the literature. It is only if both conditions of $C_{0}=1$ and $\left\langle\left\langle v_{2, \text { mix }}\right\rangle\right\rangle=0$ are met, will $S_{2,1}=0$. This coupled criteria is sometimes referred to as the critical pressure limit in the single-component multiphase flow literature, or homogenous flow in the multi-component multiphase flow literature.

\section{PROOF OF MUTUAL DEPENDENCY OF THE DRIFT FLUX PARAMETERS}

Following from Section 1.2, it can inferred from eqn (12) that there cannot be a complete mathematical dissociation between the drift flux parameters $C_{0}$ and $\left\langle\left\langle v_{2, \text { mix }}\right\rangle\right\rangle$ because they are related to each other as well as to other averaged and local velocities (and volume fractions) via this equation. We now provide an analytical proof of this mutual dependency in which we will invoke the fractional flow framework discussed in Section 1.1.

Instead of a two-parameter form, eqn (9) can be re-stated in a three-parameter form in which the averaged mixture distribution parameter, $C_{0}$, is split into two terms. The idea of splitting $C_{0}$ can also be found in prior literature, e.g. [8]. From a correlation standpoint, instead of two drift flux parameters there would now be more one more parameter available to match averaged volume fraction data. To see how $C_{0}$ can be split apart, we first analytically expand $C_{0}$ as:

$$
C_{0}=\frac{\left\langle s_{2} u_{\text {mix }}\right\rangle}{\left\langle s_{2}\right\rangle\left\langle u_{\text {mix }}\right\rangle}=\frac{\left\langle s_{2} u_{1}\right\rangle+\left\langle s_{2} u_{2}\right\rangle}{\left\langle s_{2}\right\rangle\left\langle u_{\text {mix }}\right\rangle}=\overbrace{\left(\frac{\left\langle s_{2} u_{1}\right\rangle}{\left\langle s_{2}\right\rangle\left\langle u_{1}\right\rangle}\right)}^{=C_{1}} f_{1}+\overbrace{\left(\frac{\left\langle s_{2} u_{2}\right\rangle}{\left\langle s_{2}\right\rangle\left\langle u_{2}\right\rangle}\right)}^{=C_{2}} f_{2}
$$


Where $C_{1}$ represents an averaged phase- 1 distribution parameter and $C_{2}$ represents an averaged phase-2 distribution parameter. Then, since $f_{1}=1-f_{2}$, we can simplify eqn (13) and substitute into eqn (8) to arrive at:

$$
\frac{f_{2}}{\left\langle s_{2}\right\rangle}=(\overbrace{C_{1}\left(1-f_{2}\right)+C_{2} f_{2}}^{=C_{0}}+\frac{\left\langle\left\langle v_{2, \text { mix }}\right\rangle\right\rangle}{\left\langle u_{\text {mix }}\right\rangle})
$$

We can then re-arrange eqn (14) to solve for $f_{2}$, to arrive at:

$$
\frac{f_{2}}{\left\langle s_{2}\right\rangle}=\frac{C_{1}\left\langle u_{\text {mix }}\right\rangle+\left\langle\left\langle v_{2, \text { mix }}\right\rangle\right\rangle}{\left\langle u_{\text {mix }}\right\rangle\left(1-\left\langle s_{2}\right\rangle\left(C_{2}-C_{1}\right)\right)}
$$

Putting eqn (15) into eqn (7), we get:

$$
H_{2,1}=\left(\frac{C_{1}\left\langle u_{\text {mix }}\right\rangle+\left\langle\left\langle v_{2, \text { mix }}\right\rangle\right\rangle}{\left\langle u_{\text {mix }}\right\rangle\left(1-\left\langle s_{2}\right\rangle\left(C_{2}-C_{1}\right)\right)}\right)\left(\frac{1-\left\langle s_{2}\right\rangle}{1-f_{2}}\right)
$$

Then, equating eqns (16) and (9), we get:

$$
C_{0}=\frac{C_{1}\left\langle u_{\text {mix }}\right\rangle+\left\langle\left\langle v_{2, \text { mix }}\right\rangle\right\rangle\left\langle s_{2}\right\rangle\left(C_{2}-C_{1}\right)}{\left\langle u_{\text {mix }}\right\rangle\left(1-\left\langle s_{2}\right\rangle\left(C_{2}-C_{1}\right)\right)}
$$

Equation (17) now demonstrates, in a simple, self-consistent and unambiguous way why there cannot be a dissociation between $C_{0}$ and $\left\langle\left\langle v_{2, m i x}\right\rangle\right\rangle$. This derived fact also implies that correlations for $C_{0}$ and $\left\langle\left\langle v_{2, m i x}\right\rangle\right\rangle$ in which these terms are expressed explicitly from each other cannot claim a better understanding of the flow physics unless they capture this inherent bi-directional dependency. Such explicit correlations can only claim a faithful representation of the specific data in their validation database. Indeed, it is unreasonable to suggest that such explicit correlations adhere to the flow physics, and especially so when these correlations are ad hoc constructed in mathematical form or are validated against poorly controlled published data, or worse, secret (publicly unverifiable) data of private labs.

As seen from eqn (17), all of the multiphase flow variables that affect $\left\langle\left\langle v_{2, \text { mix }}\right\rangle\right\rangle$ will also affect $C_{0}$. As an industrially relevant example such as vertical upward bubbly flow, eqn (17) now provides a mathematical justification of why a reduction in bubble drift velocity parameter (i.e. an increase in bubble residence time caused, for example, by a wall-peaking lateral void fraction profile) will lead to a reduction in the averaged mixture distribution parameter, as widely reported in the literature $[6,9,10]$. Equation (17) also provides a rational explanation for the extreme difficulty in the past to resolve the influence of the lateral distribution (multidimensional) effects of the dispersed phase in the flow (captured in an averaged way in $C_{0}$ ) on multiphase flow variables such as $\left\langle s_{2}\right\rangle,\left\langle u_{\text {mix }}\right\rangle$ and $\left\langle\left\langle v_{2, \text { mix }}\right\rangle\right\rangle$.

\section{REFORMULATING PRIOR AVERAGED VOLUME FRACTION MODELS}

Apart from reformulating drift flux equations in the fractional flow framework as shown in Section 1.2, we can also reformulate other volume fraction models from the literature in terms of slip ratios. As examples, starting from the pre-1950s era, the famous Armand [3] correlation for horizontal bubbly flow is: 


$$
H_{2,1}=(\overbrace{1.2}^{C_{0}}+\frac{\overbrace{0}^{\left\langle v_{2, m i x}\right\rangle}\rangle)}{\left\langle u_{\text {mix }}\right\rangle})\left(\frac{1-\left\langle s_{2}\right\rangle}{1-f_{2}}\right)
$$

The Butterworth [11] model can be re-cast in terms of the Lockhart and Martinelli [12] parameter, $X_{t t}$, as:

$$
H_{2,1}=0.28(\overbrace{\left(\frac{1-f_{2}}{f_{2}}\right)^{0.9}\left(\frac{\rho_{2}}{\rho_{1}}\right)^{-0.4}\left(\frac{\mu_{2}}{\mu_{1}}\right)^{-0.1}}^{X_{t t}})^{0.71}\left(\frac{1-f_{2}}{f_{2}}\right)^{-1}
$$

Where $\rho$ and $\mu$ are density and dynamic viscosity, respectively. The Fauske [13] and Zivi [14] steam-water models can be re-cast in their iso-slip form as:

$$
H_{2,1}^{\text {Fauske }}=\left(\frac{\rho_{2}}{\rho_{1}}\right)^{-0.5} \text { and } H_{2,1}^{Z i v i}=\left(\frac{\rho_{2}}{\rho_{1}}\right)^{-0.33}
$$

The Nicklin et al. [15] correlation valid for up-inclined bubbly, bubbly-slug, slug, slug-churn, churn and column flows is:

$$
H_{2,1}=(\overbrace{1.2}^{C_{0}}+\frac{\overbrace{0.35(g D)^{0.5}}^{\left\langle\left\langle v_{2, \text { mix }}\right\rangle\right\rangle}}{\left\langle u_{\text {mix }}\right\rangle})\left(\frac{1-\left\langle s_{2}\right\rangle}{1-f_{2}}\right)
$$

Where $g$ and $D$ are standard gravity and diameter, respectively. The annular flow correlation of Ishii [16] is:

$$
H_{2,1}=\left(\frac{\rho_{2}}{\rho_{1}}\right)^{-0.5}\left(\frac{\left\langle s_{2}\right\rangle^{0.5}}{1+75\left(1-\left\langle s_{2}\right\rangle\right)}\right)^{0.5}
$$

The Greskovich and Cooper [17] correlation can be reformulated as:

$$
H_{2,1}=(\overbrace{1}^{=C_{0}}+\frac{\overbrace{0.67(g D)^{0.5}(\sin (90-\theta))^{0.263}}}{\left\langle u_{\text {mix }}\right\rangle})\left(\frac{1-\left\langle v_{2}\right\rangle}{1-f_{2}}\right)
$$

The horizontal flow correlation of Garcia et al. [18] is:

$$
H_{2,1}=\frac{1}{C}\left(\frac{1-f_{2}}{f_{2}}\right)^{a-1}=K^{-1}\left(\frac{1-f_{2}}{f_{2}}\right)^{a-1}\left(\frac{\rho_{2}}{\rho_{1}}\right)^{-b}\left(\frac{\mu_{2}}{\mu_{1}}\right)^{-c}
$$

Where, 'C' and 'a' in eqn (26) are given in Table 5 of Garcia et al. [18], and, 'K', 'b' and 'c' are given in Table 1 of Chen and Spedding [19]. It is important to observe the identical mathematical form that exists between eqns (24) and (19). In viewing these seemingly different 
correlations in this unified way, it is now clear that the Garcia et al. [18] correlation is simply a re-tuned Butterworth [11] correlation with parameters that are fitted to more data. More data available for tuning, however, does not generally signify a better correlation. The sources and quality of the data matters a lot, apart from the ranges of the variables covered by the data. For example, the majority of the data (about $60 \%$ of the entire database) used to create the Garcia et al. [18] correlation are sourced from untraceable, irreproducible, and publicly inaccessible data such as the 1,255 data-points of the 'Companies' data in Table 2 of the reference.

Next, the Premoli et al. [20] correlation of the CISE (Centro Information, Studied Esperienze) group is:

$$
H_{2,1}=1+E_{1}\left(\frac{\left(\frac{f_{2}}{1-f_{2}}\right)}{1+E_{2}\left(\frac{f_{2}}{1-f_{2}}\right)}-E_{3}\left(\frac{f_{2}}{1-f_{2}}\right)\right)^{0.5}
$$

Where, ' $E_{1}$ ', ' $E_{2}$ ' and ' $E_{3}$ ' in eqn (25) are given on page 2-83 of the classic Hetsroni [21] multiphase flow handbook. Note that in viewing this correlation in this way, it now becomes clear that it will fail when the terms in the square root yield a negative value (a common occurrence in practice).

Even an expected poor performance of an averaged volume fraction model can be predetermined by reformulating them in this way. For example, the Watterson et al. [22] correlation for stratified roll waves is:

$$
H_{2,1}=0.696\left(\frac{\rho_{2}}{\rho_{1}}\right)^{-0.5}\left(\frac{1}{\left\langle u_{1}\right\rangle}\right)^{0.28}
$$

Where, eqn 26 is a dimensional correlation with tuning constants linked to a phase 1 superficial velocity, $\left\langle u_{1}\right\rangle$, in $\mathrm{m} / \mathrm{s}$. Another dimensional correlation that has been found to perform poorly is the Bankoff [4] correlation, can now be seen in its re-casted form as:

$$
H_{2,1}=\left(0.71+1.45 \times 10^{-8} P_{s y s}\right)^{-1}\left(\frac{1-\left\langle s_{2}\right\rangle}{1-f_{2}}\right)
$$

Where, the tuning constants are linked to the system pressure, $P_{s y s}$, in Pa.

Last, the Woldesmayat and Ghajar [23] correlation, valid for all inclinations and different flow patterns is eqn (9) with the drift flux parameters as:

$$
C_{0}=f_{2}\left(1+\left(\frac{1-f_{2}}{f_{2}}\right)^{\left(\frac{\rho_{2}}{\rho_{1}}\right)^{0.1}}\right)
$$

And,

$$
\left\langle\left\langle v_{2, \text { mix }}\right\rangle\right\rangle=2.9\left(\frac{g|\Delta \rho| D \sigma_{2,1}(1+\cos (90-\theta))}{\left(\rho_{1}\right)^{2}}\right)^{0.25}(1.22+1.22 \sin (90-\theta))^{\frac{P_{a m p}}{P_{s y s}}}
$$




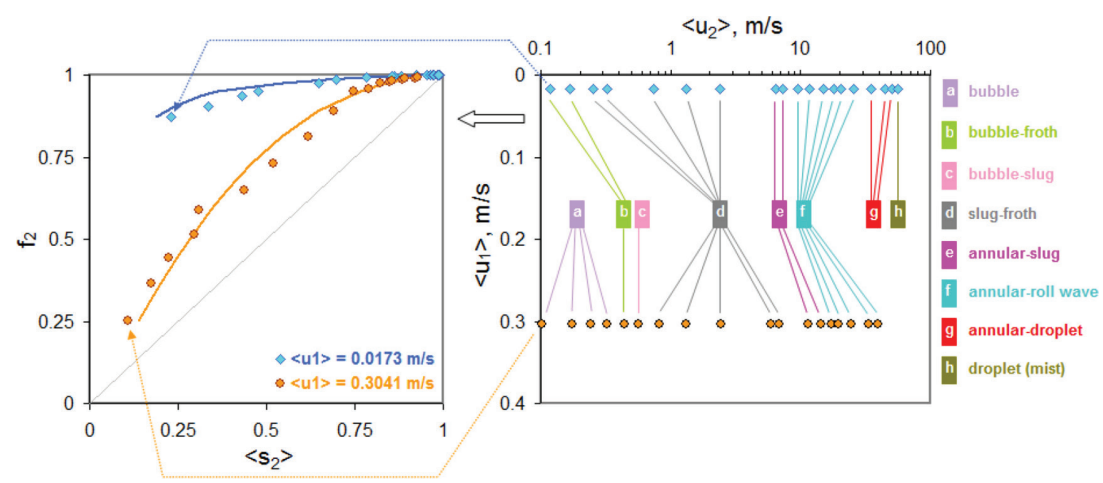

Figure 2: A graphical representation of the fractional flow paths (solid lines in left chart above) described by eqns (8), (28), and (29) as compared against two datasets [24] (diamond and circular points in the charts), spanning a wide range of flow patterns and flow rates.

Where $\sigma$ and $\theta$ are the interfacial tension and inclination angle from vertical, respectively. As a demonstration of how the $H_{2,1}$ model of Woldesmayat and Ghajar [23] describes different fractional flow paths corresponding to different datasets, Fig. 2 shows two vertical air (phase 2) and water (phase 1) datasets [24] covering the full range of flow patterns with the $H_{2,1}$ model traversing the two fractional flow paths (seen as the solid lines in the left chart) of the experimental observations.

The left chart of Fig. 2 shows how the different observed flow patterns look like when put in the dimensionless fractional flow framework. The same data when viewed in terms of dimensional flow rates are shown in the right chart of Fig. 2. In these wide-ranging experiments, runs were performed at fairly constant phase 1 superficial velocities varying from of 0.005 to $1 \mathrm{~m} / \mathrm{s}$ and increasing phase 2 superficial velocities varying from 0.1 to $58 \mathrm{~m} / \mathrm{s}$. Note that the general physical trend observed is that low liquid superficial velocities exhibit the highest averaged slip behavior and high liquid superficial velocities exhibit the lowest averaged slip behavior. Clearly, Fig. 2 shows that the $H_{2,1}$ model used in this case [23] correctly captures both the qualitative data trend as well as the quantitative averaged slip behavior of these datasets, thus demonstrating in a simple way how fractional flow paths transition through and connect different flow patterns.

\section{CONCLUSIONS}

In this contribution, the computationally efficient drift flux formulation and, more broadly, other averaged volume fraction models are reformulated in the fractional flow framework. Utilizing this fractional flow representation, it is shown via analytical derivations that the drift flux parameters cannot be disassociated from each other and are intricately related to each other as well as to the averaged and local phase velocities and volume fractions. All of the multiphase flow variables that affect the drift velocity parameter also affect the distribution parameter. Furthermore, the new analytical derivations that enable the reformulation (and unification) of drift flux and other averaged volume fraction models are used to clearly demonstrate how the fractional flow pathways in fractional flow charts transition through and connect different flow patterns, thus capturing the inter-relationships between different 
flow phenomena. As is evident, the value gained from improved understanding of the theoretical basis underlying multiphase flow simulation methods can help to explain the reasons for the match or mismatch of computing outcomes in comparison with field behaviors and observations.

\section{REFERENCES}

[1] Zuber, N. \& Findlay, J.A., Average volumetric concentration in two-phase flow systems. Journal of Heat Transfer, 87, pp. 453-468, 1965. https://doi.org/10.1115/1.3689137

[2] Chexal, B. \& Lellouche, G., Void fraction correlation for generalized applications. Progress in Nuclear Energy, 27(4), pp. 255-295, 1992. https://doi.org/10.1016/0149-1970(92)90007-P

[3] Armand, A.A., The resistance during the movement of a two-phase system in horizontal pipes. Atomic Energy Research Establishment Transactions, 828(1), pp. 16-23, 1946.

[4] Bankoff, S.G., A variable density single-fluid model two-phase flow with particular reference to steam-water. Journal of Heat Transfer, 11(Series B), pp. 265-272, 1960.

[5] Franca, F. \& Lahey, Jr., R.T., The use of drift-flux techniques for the analysis of horizontal two-phase flows International Journal of Multiphase Flow, 18(6), pp. 787-801, 1992. https://doi.org/10.1016/0301-9322(92)90059-P

[6] Guet, S. \& Ooms, G., Fluid mechanical aspects of the gas-lift technique. Annual Reviews of Fluid Mechanics, 38, pp. 255-249, 2006.

https://doi.org/10.1146/annurev.fluid.38.061505.093942

[7] Garnier, C., Lance, M. \& Marie, J.L., Measurement of local flow characteristics in buoyancy-driven bubbly flow at high void fraction. Experimental Thermal and Fluid Science, 26, pp. 811-815, 2002.

https://doi.org/10.1016/S0894-1777(02)00198-X

[8] Clark, N.N. \& Flemmer, R.L., Predicting the holdup in two-phase bubble upflow and downflow using the Zuber and Findlay Drift-Flux model. AIChE Journal, 31(3), pp. 500-503, 1985.

https://doi.org/10.1002/aic.690310323

[9] Guet, S., Ooms, G., Oliemans, R.V.A. \& Mudde, R.F., Bubble size effect on low liquid input drift-flux parameters. Chemical Engineering Science, 59, pp. 3315-3329, 2004. https://doi.org/10.1016/j.ces.2004.03.036

[10] Hibiki, T. \& Ishii, M., Distribution parameter and drift velocity of drift-flux model in bubbly flow. International Journal of Heat and Mass Transfer, 45, pp. 707-721, 2002. https://doi.org/10.1016/S0017-9310(01)00195-8

[11] Butterworth, D., A comparison of some void-fraction relationships for co-current gasliquid flow. International Journal of Multiphase Flow, 1, pp. 845-850, 1975. https://doi.org/10.1016/0301-9322(75)90038-5

[12] Lockhart, R.W. \& Martinelli, R.C., Proposed correlation of data for isothermal twophase, two- component flow in pipes. Journal of Chemical Engineering Progress, 45(1), pp. 39-48, 1949.

[13] Fauske, H., Critical two-phase, steam-water flows. Proceeding of the 1961 Heat Transfer and Fluid Mechanics Institute, Stanford University Press, 1961.

[14] Zivi, S.M., Estimation of steady state steam void fraction by means of the principle of minimum entropy production. Journal of Heat Transfer, 86, pp. 247-252, 1964. https://doi.org/10.1115/1.3687113 
[15] Nicklin, D.J., Wilkes, J.O. \& Davidson, J.F., Two-phase flow in vertical tubes. Transaction of Institution Chemical Engineers, 40(61), 1962.

[16] Ishii, M., Thermo-Fluid Dynamic Theory of Two-Phase Flow, Eyrolles, Paris, 1975.

[17] Greskovich, E.J. \& Cooper, W.T., Correlation and prediction of gas liquid holdups in inclined upflows. AIChE Journal, 21, pp. 1189-1192, 1975. https://doi.org/10.1002/aic.690210619

[18] Garcia, F., Garcia, R. \& Joseph, D.D., Composite power law holdup correlations in horizontal pipes. International Journal of Multiphase Flow, 31, pp. 1276-1303, 2005. https://doi.org/10.1016/j.ijmultiphaseflow.2005.07.007

[19] Chen, J.J. \& Spedding, P.L., An analysis of holdup in horizontal two-phase gas liquid flow. International Journal of Multiphase Flow, 2, pp. 147-159, 1983. https://doi.org/10.1016/0301-9322(83)90049-6

[20] Premoli, A., Francesco, D. \& Prima, A., An empirical correlation for evaluating twophase mixture density under adiabatic conditions. European Two-Phase Flow Group Meeting, Milan, Italy, 1970.

[21] Hetsroni, G., Handbook of Multiphase Systems, Hemisphere Publ. Co., 1982.

[22] Watterson, J.K., Cooper, R.K. \& Spedding, P.L., Prediction of pressure loss and holdup in two-phase horizontal stratified roll-wave pipe flow. Industrial \& Engineering Chemistry Research, 41, pp. 6621-6622, 2002. https://doi.org/10.1021/ie010749y

[23] Woldesmayat, M.A. \& Ghajar, A.J., Comparison of void fraction correlations for different flow patterns in horizontal and upward inclined pipes. Internatioanal Journal of Multiphase Flow, 33, pp. 347-370, 2007.

https://doi.org/10.1016/j.ijmultiphaseflow.2006.09.004

[24] Spedding, P.L. \& Nguyen, V.T., Data on holdup, pressure loss and flow pattern for twophase, air-water flow in an inclined pipe. Report 122, U. of Auckland, Auckland, New Zealand, 1976. 